\title{
Leakage of photospheric acoustic waves into non-magnetic solar atmosphere
}

\author{
R. Erdélyi ${ }^{1}$, C. Malins ${ }^{1}$, G. Tóth ${ }^{2}$, and B. De Pontieu ${ }^{3}$ \\ ${ }^{1}$ Solar Physics and Space Plasma Research Centre (SP $\left.{ }^{2} \mathrm{RC}\right)$, Department of Applied Mathematics, University of Sheffield, The Hicks \\ Building, Hounsfield Road, Sheffield S3 7RH, UK \\ e-mail: [robertus;c.malins]@sheffield.ac.uk \\ 2 Department of Atomic Physics, Eötvös Loránd University, Pázmány Péter sétány 1/A, Budapest 1117, Hungary \\ e-mail: gtoth@umich.edu \\ 3 Lockheed Martin Solar and Astrophysics Laboratory, 3251 Hanover Street, Org. ADBS, Building 252, Palo Alto, CA 94304n, USA \\ e-mail: bdp@lmsal.com
}

Received 1 December 2006 / Accepted 3 March 2007

\begin{abstract}
Aims. This paper aims to look at the propagation of synthetic photospheric oscillations from a point source into a two-dimensional non-magnetic solar atmosphere. It takes a particular interest in the leakage of 5-min global oscillations into the atmosphere, and aims to complement efforts on the driving of chromospheric dynamics (e.g. spicules and waves) by 5-min oscillations.

Methods. A model solar atmosphere is constructed based on realistic temperature and gravitational stratification. The response of this atmosphere to a wide range of adiabatic periodic velocity drivers is numerically investigated in the hydrodynamic approximation. Results. The findings of this modelling are threefold. Firstly, high-frequency waves are shown to propagate from the lower atmosphere across the transition region experiencing relatively low reflection and transmitting energy into the corona. Secondly, it is demonstrated that driving the upper solar photosphere with a harmonic piston driver at around the 5 min period may generate three separate standing modes with similar periods in the chromosphere and transition region. In the cavity formed by the chromosphere and bounded by regions of low cut-off period at the photospheric temperature minimum and the transition region this is caused by reflection, while at either end of this region in the lower chromosphere and transition region the standing modes are caused by resonant excitation. Finally, the transition region becomes a guide for horizontally propagating surface waves for a wide range of driver periods, and in particular at those periods which support chromospheric standing waves. Crucially, these findings are the results of a combination of a chromospheric cavity and resonant excitation in the lower atmosphere and transition region.
\end{abstract}

Key words. hydrodynamics - methods: numerical - Sun: chromosphere - Sun: oscillations - Sun: atmosphere Sun: transition region

\section{Introduction}

The solar photosphere is subjected to a constant buffeting and movement from the solar surface driven by a great range of physical mechanisms. These buffeting motions (including periodic and random motions) are driven by local effects such as sub-photospheric turbulence in the convection zone, and also by global effects such as the solar global $p$-modes. Whatever the source of these motions, there is the potential for them to generate propagating disturbances, both into chromospheric magnetic network regions at the boundaries of supergranulation cells and into the relatively non-magnetic internetwork regions. Carlsson \& Stein (1992) examined the generation of Ca K2V bright points by upwards propagating acoustic shocks in the solar chromosphere, demonstrating that a sinusoidal $180 \mathrm{~s}$ period driver was able to produce observed results well. Recent observational work by Marsh \& Walsh (2006) has provided evidence of the propagation of oscillatory signals from the transition region into the solar corona in the 3-min period band, and it has been suggested in the past that 3-min oscillations observed in the transition region are connected to the 5-min global p-modes (Bogdan 2000; Zhukov 2002). Leibacher et al. (1982) suggest that the observed chromospheric modes are the result of a chromospheric cavity, while Fleck \& Schmitz (1991) contend that a cavity is unnecessary to explain the chromospheric oscillations, and that the correct explanation is the resonant excitation of the cut-off frequency at the temperature minimum. The numerical simulation of the propagation of oscillatory signals at these periods from the photosphere into the corona is therefore an area of great interest and relevance.

A great deal of work has been carried out examining the propagation of photospheric signals in regions of strong magnetic field where waves are to some extent guided by field lines (Bogdan et al. 2003; De Pontieu et al. 2004; Hasan et al. 2005). However, it is also instructive to examine the non-magnetic case using numerical tools, in order to look for a better understanding of purely acoustic effects, and as an approximation to the quiet internetwork Sun. Fleck \& Schmitz (1991) have performed numerical simulations in a one dimensional linear regime, in both an isothermal case and the case of the VAL IIIc atmosphere, while Carlsson \& Stein (1992) have used nonLTE radiative hydrodynamics in the VALIIIc atmosphere. Here two-dimensional non-linear simulations, including the transition 
region and corona, but not including radiative and non-LTE effects are presented.

\section{Equilibrium}

In this work, the propagation of driven acoustic waves in a nonmagnetic stratified two-dimensional solar atmosphere has been addressed. Previous studies have examined the effect of gravitational and temperature stratification on wave propagation in the solar corona (De Moortel \& Hood 2003, 2004) and in a two isothermal layer model of the photosphere, chromosphere and corona (Chaouche \& Abdelatif 2005) and demonstrated that gravitational and temperature stratification is crucial in understanding propagation of slow waves, which are analogous to pure acoustic waves, through the solar atmosphere. Also, work by O'Shea et al. (2006) has found observational evidence not only for outwardly propagating slow magnetoacoustic waves (in off-limb polar regions) but also have found evidence that such propagating waves are influenced by passage through some sort of resonant cavity.

It is therefore considered important that the model atmosphere used in the simulations is based closely upon accurate observationally derived models of the structure of the solar atmosphere, and that the simulations therefore fully include the effects on wave propagation of realistic atmospheric gravitational and temperature stratification. Unfortunately, there has been some contention in recent years as to what the temperature structure of the solar chromosphere actually is (Carlsson \& Stein 1995; Ayres 1998, 2002; Kalkofen et al. 1999; Kalkofen 2001). The VALIIIc (Vernazza et al. 1981) quiet Sun model which has been extensively used in the past as a basis for quiet Sun modelling has been called into question by the work of Carlsson \& Stein (1995) which suggests strongly that the existence of a chromospheric temperature rise is chimerical, and that the chromosphere is in fact a more dynamic region which spends most of the time in a relatively cold state with occasional shock heating producing the appearance of a temperature increase under the type of analysis which is used to produce such semi-empirical models as VAL. Kalkofen (2001) has argued the counterpoint to this case, recognising that the chromosphere should be considered dynamic but arguing that there is still evidence of sustained chromospheric heating. Ayres (2002) on the other hand points out that there is evidence from $\mathrm{CO}$ lines that the chromosphere has typical temperatures which are actually below those suggested by Carlsson \& Stein (1995). Avrett (1996) recognises that the next generation of model stellar atmospheres needs to address the questions raised by the increasing sophistication of numerical modelling and observations which have allowed the gaps in previous models to be exposed.

This all begs the question of what is an appropriate model to use in the numerical simulations of the non-magnetic solar chromosphere. The VAL IIIc model has been used as the basis for the chromosphere for the bulk of the work presented here, which gives an added advantage of increasing comparibility with previous work based on this model. However, in recognition of the importance of the cold chromospheric hypothesis the key results (those using a $300 \mathrm{~s}$ harmonic driver) have been reproduced using a model with an isothermal chromosphere at $\sim 4400 \mathrm{~K}$. A negative temperature gradient as is predicted by Carlsson \& Stein (1995) would introduce problems of convective instability which it was desired to avoid.

The lower part of the initial profile of the first equilibrium implemented here is based on the VAL IIIc (Vernazza et al. 1981) atmospheric model. The temperature-height distribution in VAL IIIc is constructed to provide as close a match as possible to observed solar emission spectra, and this temperature profile is the starting point in constructing a model atmosphere. There are, however, some differences between the method used in the construction of the VAL IIIc atmosphere and that which is being used to advect the model here. In particular, the VAL model includes a turbulent pressure term based on the existence of a varying microvelocity $V$. The numerical model used here does not include this term, and thus the temperature profile is an exact match to the VAL IIIc atmosphere while the pressure and density profiles are qualitatively similar. Although the pressure and density depart somewhat from the VAL IIIc values, the sound speed is the key parameter in determining the behaviour of driven propagating acoustic waves, and this only depends on the temperature. The lower part of the second equilibrium investigated is set to be isothermal up to the transition region with a temperature of $4400 \mathrm{~K}$.

The solar atmosphere is not described in VAL IIIc above $2.5 \mathrm{Mm}$, and therefore the upper part of the atmosphere is constructed based on McWhirter et al. (1975). In the same way as with the VAL IIIc based lower atmosphere, the temperature profile has been taken, adjusting the McWhirter et al. (1975) height scheme to be consistent with VAL IIIc, while the density and pressure are derived from this temperature profile and the condition of hydrostatic equilibrium.

VAL IIIc includes a temperature plateau in the transition region, on which the otherwise steep transition region temperature gradient drops towards zero. It is computationally challenging to maintain this feature in hydrostatic equilibrium, and more importantly Fontenla et al. (1990) demonstrate that the inclusion of ambipolar diffusion in semi-empirical temperature modelling removes the need to include such a plateau. Therefore it has been smoothed out in the process of linking the VAL IIIc and McWhirter et al. (1975) temperature profiles. The atmosphere is only being studied above the photospheric temperature minimum, in order to eliminate convective instabilities from the computations, as these could obscure the behaviour of the propagating waves. Much of the photosphere in which $300 \mathrm{~s}$ waves are evanescent therefore does not feature in the simulations.

Mechanisms for leakage into the photosphere and chromosphere of these lower frequency waves have been studied by De Pontieu et al. (2004) in 1D field aligned (M)HD, who suggest that in the presence of a non-vertical magnetic field the region of evanescence may be adequately reduced to allow significant wave leakage through the temperature minimum in a one-dimensional MHD waveguide. The work here does not include any such magnetic wave guides, however it is shown that signals are still able to penetrate into the chromosphere and the corona in the regime being modelled. The addition of appropriate magnetic field geometries is left for future papers, but is expected in some configurations to enhance the leakage of driven waves and hence energy into the middle and upper atmosphere as pointed out by De Pontieu et al. $(2004,2005)$ in their 1D studies.

The initial equilibrium atmosphere is constructed from the above detailed temperature profiles according to the condition of hydrostatic equilibrium

$$
\frac{\mathrm{d} p}{\mathrm{~d} z}=-\rho g
$$

where $p$ is the kinetic gas pressure, $z$ is vertical distance from the base of the atmosphere, $\rho$ is mass density and $g$ is gravitational acceleration. 
From the condition of hydrostatic equilibrium and the assumption of an ideal gas

$\rho=\frac{m p}{k_{\mathrm{B}} T}$,

(for $m$ the mean particle mass, $T$ temperature and $k_{\mathrm{B}}$ Boltzmann's constant) are derived relations for pressure and density

$p(z)=p_{0} \exp -\left(\int_{0}^{z} \frac{1}{\Lambda(z)} \mathrm{d} z\right)$,

$\rho(z)=\rho_{0} \frac{T_{0}}{T(z)} \exp -\left(\int_{0}^{z} \frac{1}{\Lambda(z)} \mathrm{d} z\right)$,

where $\Lambda$ is the pressure scale height

$\Lambda(z)=\frac{k_{\mathrm{B}} T(z)}{m g}$.

The stratification is thus completely determined by the semiempirical temperature profile and the boundary parameter $\rho_{0}$.

Low gravity regions (De Pontieu 1996; James 2004) are implemented at the higher and lower extents of the computational domain in order to prevent downflows from developing with the use of open boundaries. Data from within these low gravity regions are considered unphysical and are not included in the analysis. The lower and upper boundaries are open to prevent reflection. The upper boundary is in the corona, and any reflections at this point would be physically misleading. Except in these regions, it is a reasonable simplification to treat gravity as a constant over the domain which extends for only $\sim 1 \%$ of a solar radius. Gravity is taken as $274 \mathrm{~m} \mathrm{~s}^{-2}$.

\section{Numerical method}

The numerical simulations are performed using the Versatile Advection Code (Tóth 1996). This code is used to solve the full system of ideal hydrodynamic equations listed below in twodimensional cartesian geometry. A Total Variation Diminishing - Lax-Friedrichs spatial discretization scheme with a Woodward limiter is used as implemented in the VAC, and a time discretisation based on a Courant parameter of 0.8 . The simulation domain is $8 \mathrm{Mm}$ wide and $8 \mathrm{Mm}$ high, with a resolution of $200 \times 510$ grid points plus boundary layers at each edge two grid points wide. The grid points are positioned on a mesh that is vertically stretched, with highest resolution at the transition region, and significantly lower resolution in the corona. The areas of higher resolution match those areas of the domain which are associated with the highest gradients. The mesh is not adaptive in time, but the area of highest gradient (i.e. the transition region) does not move significantly in space in any of the simulations.

The lower extent of the computational domain corresponds to the solar photosphere, with the chromosphere above in the region $\sim 0.5-2 \mathrm{Mm}$ height. The transition region is positioned from approximately 2-3 Mm height, and the corona extends to about $7 \mathrm{Mm}$ with the final $1 \mathrm{Mm}$ of the domain containing the upper low gravity region.

All of the boundary layers are given boundary conditions of the form $\mathrm{d} u_{j} / \mathrm{d} x_{i} \equiv 0$, where $u_{j}$ are the flow variables and $i$ indexes the direction to which the boundary is perpendicular. These boundary conditions allow disturbances to propagate out of the computational domain with negligible reflection in the simulations described here.

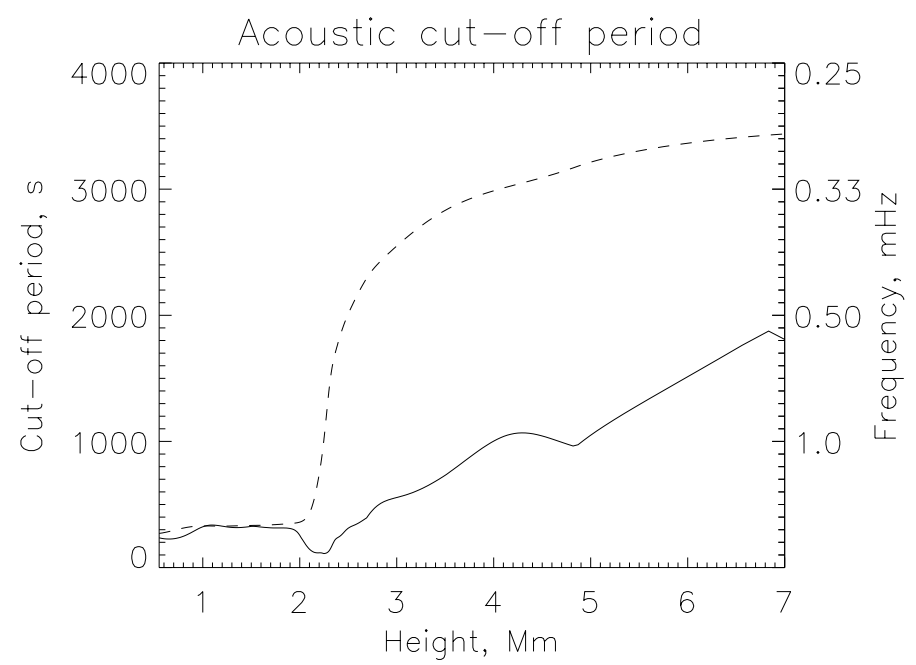

Fig. 1. Variation in cut-off period with changing height ignoring stratification (dashed line) and including stratification (solid line).

\subsection{Equations}

The equations of hydrodynamics read as follows

$$
\begin{aligned}
\partial_{t} \rho+\nabla \cdot(\boldsymbol{v} \rho) & =0 \\
\partial_{t}(\rho \boldsymbol{v})+\nabla \cdot(\boldsymbol{v} \rho \boldsymbol{v})+\nabla p & =\boldsymbol{F}_{\mathrm{g}} \\
\partial_{t} e+\nabla \cdot(\boldsymbol{v} e+\boldsymbol{v} p) & =0 \\
p & =(\gamma-1)\left(e-\rho \boldsymbol{v}^{2} / 2\right)
\end{aligned}
$$

where $\boldsymbol{v}$ is velocity, $\boldsymbol{F}_{\mathrm{g}}$ is the force due to gravity and $e$ is the internal energy. These are advected using the VAC discretisation.

\section{Simulations}

Simulations have been performed perturbing the equilibrium atmosphere at a range of driver periods from 30 to $450 \mathrm{~s}$. The periods around $300 \mathrm{~s}$ are of particular interest because they correspond to the peak of acoustic power observed at $5 \min p$-mode oscillations in the photosphere.

An important parameter in models of the propagation of photospheric disturbances into the upper atmosphere is the chromospheric local acoustic cut-off frequency. For a simple isothermal atmosphere the cut-off frequency for waves propagating vertically is

$f_{\mathrm{a}}=\frac{\gamma g}{4 \pi c_{\mathrm{s}}}$

which corresponds to a local cut-off period of

$P_{\mathrm{a}}=\frac{1}{f_{\mathrm{a}}}$

Applying this formula to the VALIIIc based temperature profile used here gives the dashed line in Fig. 1, which is uniformly increasing from the temperature minimum in line with the temperature.

In this study, however, the atmosphere is not isothermal, with particularly strong temperature gradients in the transition region which is the region of interest to us. The specification of a single value for the cut-off frequency of an atmosphere stratified in density, pressure and temperature, and under gravity, is not a trivial exercise. There are various different formulations of the cut-off frequency presented in the literature, several of which are 
compared by Schmitz \& Fleck (1998). The equation of vertical Lagrangian displacement can be manipulated for an ideal gas to give a relation for the cut-off angular frequency $\omega_{\mathrm{a}}$

$\omega_{\mathrm{a}}^{2}=\frac{\gamma^{2} g^{2}}{4 c_{\mathrm{s}}^{2}}+\frac{\gamma g}{2 c_{\mathrm{s}}^{2}} \frac{\mathrm{d} c_{\mathrm{s}}^{2}}{\mathrm{~d} z}$

This is equivalent to the simpler formulation presented by e.g. (Roberts 2004)

$\omega_{\mathrm{a}}=\frac{\gamma g}{2 c_{\mathrm{s}}} \sqrt{1+2 \Lambda^{\prime}}$

where $\Lambda^{\prime}$ is the $z$ derivative of the pressure scaleheight (see Eq. (4)). From the frequency the cut-off period can easily be derived, which is found to include local minima (see the solid line in Fig. 1). The isothermal cut-off period is included in Fig. 1 for comparison and to emphasise the importance of stratification in considering this region of the Sun.

This is not, however, the only cut-off frequency which can be calculated. By considering the Lagrangian pressure perturbation and transforming the independent and dependent variables, it is possible to find at least three additional formulations of the cut-off frequency. Schmitz \& Fleck (1998) show that these all give non-negligible variations in the cut-off frequency when applied to the the VALIIIc lower atmosphere. Here the form presented above in Eq. (12) is referred to whenever the cut-off is mentioned, with two justifications. Firstly, the other forms all include terms in the second derivative of $c_{\mathrm{s}}{ }^{2}$ (equivalently the second derivative of temperature or pressure scaleheight). Schmitz $\&$ Fleck (1998) point out that these second derivatives cannot be calculated in the transition region without giving unlimited uncertainties. Secondly, although it is certainly the case that there are significant differences between the cut-off frequencies suggested by the different forms, they should all share the key features which are relevant to the work here. Specifically, they are very similar in the lower atmosphere where the temperature rise is approximately linear, all predict a high cut-off period in the corona and all predict a drop in cut-off period due to stratification at the transition region. Provided the value of the cut-off provided by Eq. (12) is regarded as an indicator rather than as an exact value at which wave behaviour is completely transformed, it remains a key parameter for analysis of the results of the simulations.

The case in which there is an isothermal chromosphere is very similar to the VALIIIc case, but with a cut-off period in the photosphere and chromosphere which is constant and a little below $300 \mathrm{~s}$ at $\sim 270 \mathrm{~s}$, and of course does not have a temperature minimum and therefore has no corresponding minimum in cutoff period.

Although the cut-off frequency varies to some extent on small scales, the atmosphere may be divided into four distinct key regions for the purposes of analysing the results: first the photosphere around the temperature minimum, in which the cutoff period is below $300 \mathrm{~s}$ and thus $5 \mathrm{~min}$ oscillations will be evanescent; second the chromosphere in which the cut-off period increases to a value slightly above $300 \mathrm{~s}$, and therefore any signal of period 5 min or less will propagate; thirdly a zone around the transition region in which the cut-off period drops to a much lower value, perhaps as low as $100 \mathrm{~s}$, over a height range of about $400 \mathrm{~km}$; fourthly the corona in which the cut-off period again becomes sufficiently high that waves at all of the periods being considered here will propagate freely.

Lamb (1932) demonstrated that the propagation of acoustic waves in an unbounded medium was affected by the acoustic cut-off period. This work has been extended by, among others Fleck \& Schmitz (1991) and Sutmann et al. (1998) who have examined analytically the isothermal case under various types of disturbances. Crucially, acoustic waves in a gravitationally stratified atmosphere will only propagate if the period is less than the acoustic cut-off period in the atmosphere, whereas any waves with higher periods will be evanescent in these areas. In addition to this, Lamb demonstrated that the cut-off frequency is the natural frequency of the atmosphere and that any acoustic disturbances imposed on such an atmosphere will trigger a response at the cut-off period. The linear theory for a propagating pulse (Rae \& Roberts 1982) leads us to expect that a pulse travelling in a stratified atmosphere should generate a wake excitation at the natural acoustic cut-off period. It is found (Fleck \& Schmitz 1991; Kalkofen et al. 1994; Sutmann et al. 1998) that harmonic excitation will also generate a response at the cut-off period. In the case of a piston driver above the cut-off period, Fleck \& Schmitz (1991); Kalkofen et al. (1994) showed in an isothermal case that the cut-off period oscillation has a higher growth rate due to stratification (De Moortel \& Hood 2004) than the driven signal, and thus at sufficient heights comes to dominate. This conforms to the expectation that an evanescent signal will be damped with height. In the case of a driver with period below the cut-off period, Kalkofen et al. (1994) found that a signal at the cut-off frequency is generated and that it experiences stratification driven growth at the same rate as the driven signal, but that the driven signal dominates at all heights. Physically, it can be interpreted that evanescent waves transfer significant energy to modes at the cut-off frequency, whereas the propagating waves transfer little energy to cut-off frequency modes which are excited primarily by the initial pulse, and therefore the cutoff frequency oscillations will have much lower amplitude.

From Fig. 1 it is apparent that the cut-off period rises from $\sim 300 \mathrm{~s}$ in the chromosphere to $>1500 \mathrm{~s}$ in the corona. However, in the transition region because of the strong temperature stratification it drops to $<150 \mathrm{~s}$. This drop is of great importance to the study of propagation through the transition region, and demonstrates the importance of using realistic temperature profiling if the transition region is to be properly modelled. Also, at $200 \mathrm{~km}$ (where the $300 \mathrm{~s}$ driver is applied), the cut-off period is below $300 \mathrm{~s}$, and the minimum cut-off period that signals pass through in the photosphere is $\sim 210 \mathrm{~s}$. The important 5-min oscillations are therefore evanescent in the photosphere but could tunnel through it to reach the chromosphere. This tunneling occurs for the oscillation to traverse the distance from the driver application height to the propagative region.

The lower atmospheric and coronal values of the cut-off frequency are adequately high that there is no potential for signals to be evanescent in the corona, and as mentioned above signals oscillating below the cut-off period generate only insignificant amplitudes of resonant excitation at the cut-off.

Partial reflection of acoustic waves at all frequencies is expected at the transition region, with frequency dependent coefficient of reflection, as it exhibits sufficiently large gradients in density and pressure that it behaves somewhat like a density discontinuity, with lower sound speed below and higher sound speed above. This delineates the upper boundary of the chromospheric cavity which has been previously suggested as the source of 3-min transition region oscillations (Leibacher et al. 1982).

It is therefore expected that acoustic-gravity waves with periods below the transition region cut-off should propagate through the chromosphere and into the corona with relatively little energy being transferred to the cut-off frequency resonant mode, but some fraction of the wave energy being trapped by 
reflection in the chromospheric cavity. In contrast, waves with periods above this limit are expected to be evanescent as they traverse the transition region, and to generate a strong response in the transition region at the cut-off period, which will reduce energy transmission into the corona. For particularly low $(<4 \mathrm{mHz})$ frequencies the signal will experience evanescence in the photosphere at the driver position, in which case the resonantly excited mode is likely to become dominant, and potentially for the lowest $(<3 \mathrm{mHz})$ the signal may also be evanescent throughout the chromosphere. For these very high period signals, the cut-off period would be expected to be dominant at all heights.

The analytical work (Fleck \& Schmitz 1991) predicts that as the time tends to infinity in a simple atmosphere the driver period will become dominant. The photospheric $p$-modes being considered here are normally coherent over about 5 or 6 periods $(<1800 \mathrm{~s})$. It is not immediately clear whether this represents a long enough time for the driver frequency to become dominant in the simulations.

\section{High frequency}

\subsection{0 s driver}

It is expected that acoustic waves with high frequencies $(10-50 \mathrm{mHz})$ will be produced in subsurface layers of the Sun (Fossum \& Carlsson 2005) and it has been suggested in the past that they may constitute the dominant heating mechanism for the chromosphere. Fossum \& Carlsson (2005) demonstrate, using radiative $1 \mathrm{D}$ numerical hydrodynamic simulation, that the energy flux of such waves is not sufficient to account for radiative losses in the chromosphere. High frequency waves are also strongly radiatively damped in the upper photosphere (Carlsson $\&$ Stein 2002) which makes the study of their propagation into the upper solar atmosphere relatively academic. Nevertheless, the simulation of the propagation of high frequency waves into the chromosphere and corona is of interest from a diagnostic perspective, and working in 2D it becomes possible to examine the effects of attenuation on propagating high frequency waves, although it is important to note that without the radiative losses which dominate high frequency waves this study is only an indicator.

Drivers at several periods in the high frequency range were studied, with the drivers applied just above the temperature minimum at a height of $450 \mathrm{~km}$. The case of a $30 \mathrm{~s}$ driver is discussed, but the main conclusions apply equally well to other drivers with periods well below the cut-off period, and $30 \mathrm{~s}$ is of course well below the local acoustic cut-off period at any point. Figure 2 is a typical snapshot of the vertical velocity, after some time has passed. On the 2D image on the left, the spherically propagating wavefronts are clearly apparent in the lower atmosphere, while in the corona the enhanced wave speed distorts the shape of the wavefronts and causes the increased spatial separation.

In the snapshot of the vertical velocity perturbation in Fig. 2, it is apparent that the amplitude of the velocity perturbations increases with height up to the transition region. This is a result of the gravitational and temperature stratification of the atmosphere, as expected (De Moortel \& Hood 2004). This amplitude growth shows that the stratification driven amplification is stronger than the attenuation of the wave front during propagation. In the presence of a magnetic field acting as a wave guide, this growth would be even more pronounced for any slow/mixed modes which share some of the characteristics of these acousticgravity modes, and can be considered analagous in behaviour (De Pontieu et al. 2004).
The transit of the waves across the high gradients at the transition region into the corona causes the amplitude of the perturbation to be most rapidly enhanced, due to the high temperature stratification in this region. The waves then propagate away through the corona, with the amplitude slowly decreasing because the atmospheric stratification here is insufficient to counter the natural dispersal of the wave. Of course, the higher coronal temperature is accompanied by a larger sound speed, and therefore there is a significant increase in the propagation speed of the wave as it passes the transition region. These results are all qualitatively consistent with the results presented by (De Moortel \& Hood 2004) for the vertical propagation of perturbations guided by a magnetic field in a gravitationally stratified one-dimensional isothermal atmosphere. In a regime which included thermal conduction, the high period waves which reach the corona would be strongly damped.

A useful way to visualise wave propagation at a given height is to construct time-distance images. Because the sound speed is uniformly increasing with height in the VALIIIc atmosphere, it is possible with a little care to infer the direction of propagation of any of the wavefronts which appear on these images. Because the waves are emitted by a point source as spherical wavefronts, they will naturally take longer to reach the horizontal extrema of the domain than the centre at any given height. Even in an isothermal atmosphere with constant sound speed, this will give a wave travelling up from the source a curvature like this $\smile$, especially at the centre of the wavefront. In addition, an upwardly propagating front will be accelerating as it propagates, and this will tend to accentuate the upwards curvature of the wavefront in the time-distance representation even more. Downwardly propagating waves on the other hand are propagating from the line of reflection rather than a single point and decelerate as they travel, so that the wave fronts will appear flatter and with curvature slightly downwards, something more like this $\vee$. Any wavefronts propagating purely horizontally will move at the sound speed for the height in question and have a straight line signature of constant gradient, where the gradient will match the sound speed. Figure 3 a shows the signal at a height of $1.3 \mathrm{Mm}$. The first few wavefronts pass through showing curvature of a wave propagating upwards. After about $240 \mathrm{~s}$ the first reflected wavefront reaches this height. These reflected waves are travelling down with reducing sound speed, and thus display the opposite curvature. It is clear from the time-distance image that the reflected component of the wave has a somewhat smaller amplitude than the incident wave. However, it is also spread over a larger area, and it is estimated that approximately $40 \%$ of the wave energy is transmitted into the corona while the other $60 \%$ is trapped in the lower atmosphere by the reflection.

The waves exhibit the highest amplitudes (visible here as the lightest and darkest regions) directly above the driver. This is because where the wave is travelling vertically, the stratification induced amplification is most significant (see Fig. 2), and the distance travelled by the wavefront is least. Further out the signals have a much lower amplitude. The reflected wavefronts in contrast have approximately similar amplitudes from the centre to the horizontal extent of the domain, which suggests that the more inclined propagation at the edges of the box leads to stronger reflection, and the changing sound speed refracts them so that the direction of propagation is primarily vertically downwards.

The time distance image for a height of $3.3 \mathrm{Mm}$ in Fig. 3b has wavefronts that are much flatter, indicative of the higher coronal sound speed. The small curvature which is apparent shows that these signals are propagating upwards. At the 

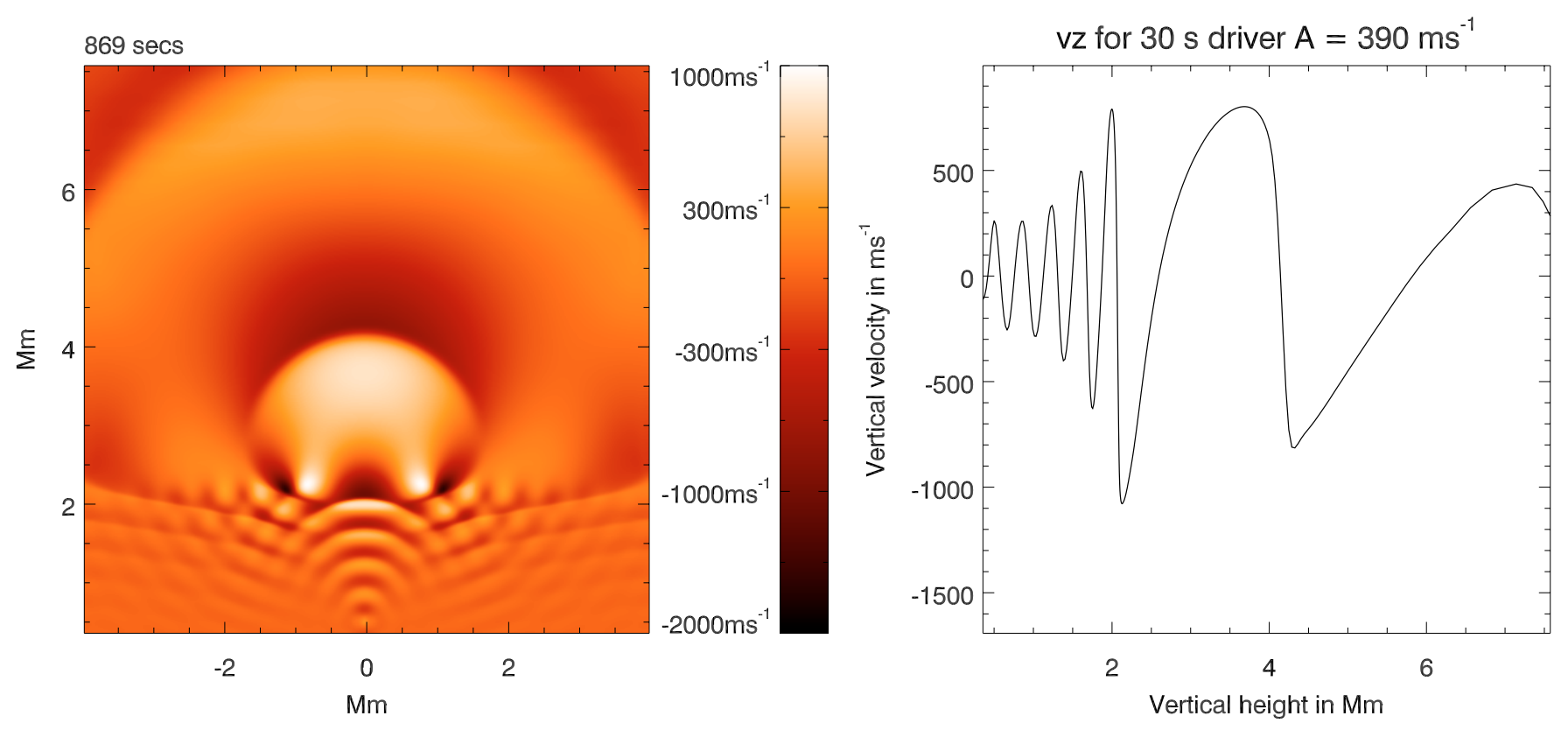

Fig. 2. Typical snapshot of the vertical velocity in the model for the $30 \mathrm{~s}$ driver, taken after $869 \mathrm{~s}$. On the left is a visualisation of the full domain, accompanied on the right by a plot taken along a vertical cross-section at the centre of the domain.
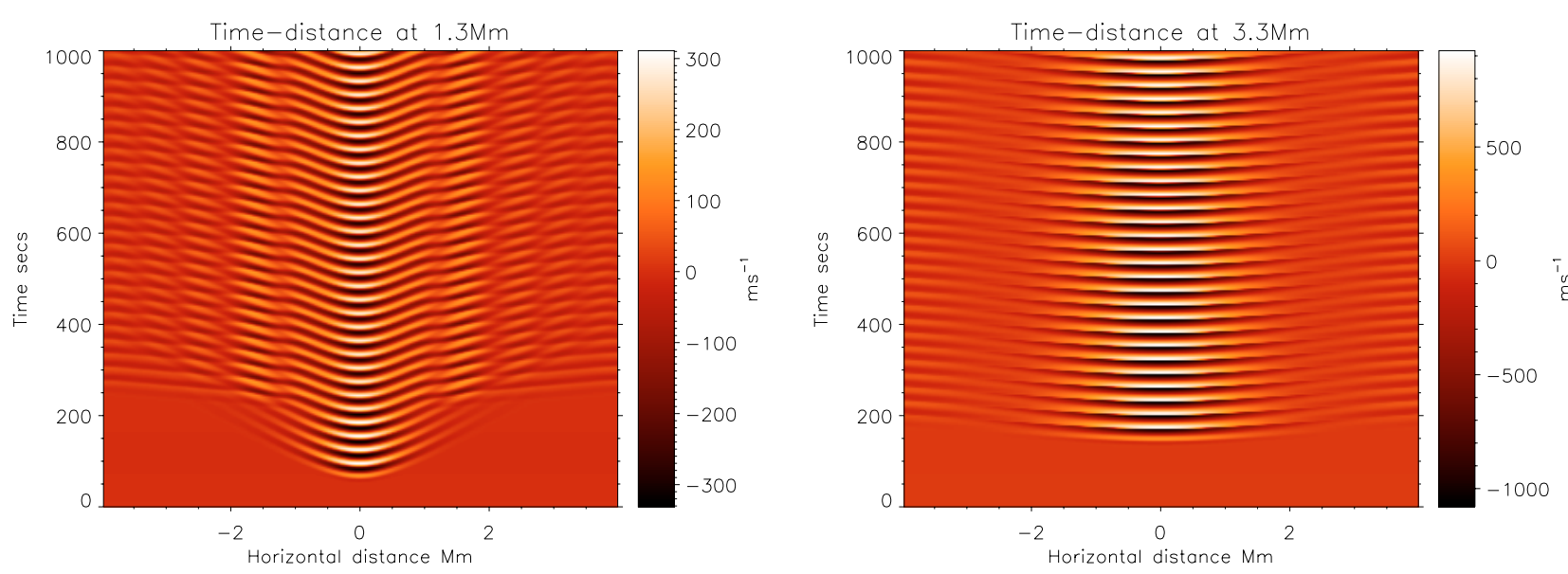

Fig. 3. a) Time-distance image for $30 \mathrm{~s}$ driver showing waves moving across a line taken at $1.3 \mathrm{Mm}$ into the atmosphere (in the chromosphere). b) Similar to a) but taken at a height of $3.3 \mathrm{Mm}$ and thus in the corona.

horizontal extrema of the domain there is evidence of a slightly different element to the signal with the curvature indicative of downwards propagation. Examining Fig. 2 at the height of 3.3 Mm the explanation is clear. At a sufficient horizontal distance from the centre of the domain, the $3.3 \mathrm{Mm}$ line is below the height at which the wavefront achieves its maximum width and is propagating horizontally, and the signal actually has a small downwards component. There is no reflection coming from higher in the atmosphere, and no interference with the cleanly propagating signal is apparent.

The data from the $30 \mathrm{~s}$ driver does not show any clear evidence of the generation of a resonant signal at the cut-off period, and the use of wavelet analysis does not show any clear evidence of strong power in the high period range that is covered by the cut-off at various heights. This is all consistent with the prediction that any driven signals below the cut-off period would dominate any resonant response.
The signal is associated with some temperature variations, but these are insignificant compared to the background temperature. In particular, at a driven amplitude of $390 \mathrm{~m} \mathrm{~s}^{-1}$, the waves do not drive any significant change in the position of the transition region nor do they drive any significant flow of cool matter into the corona.

\section{Low frequency}

\subsection{0 s driver}

The $300 \mathrm{~s}$ driver is of particular interest because this is around the period of many oscillations observed in coronal loops with footpoints in plage regions (De Moortel et al. 2002a,b), and of oscillations in transition region moss (De Pontieu et al. 2003, 2005), and the dominant $p$-mode period in the photosphere. The simulations model the potential propagation of 5-min acoustic signals in the non-magnetic atmosphere from the photosphere 


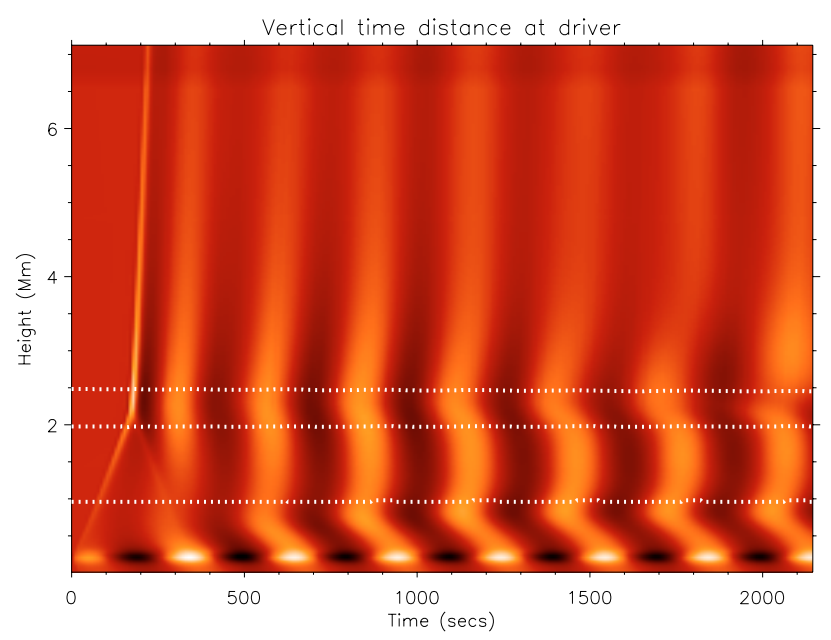

Fig. 4. Time distance image along the central vertical axis of the computational domain showing vertical velocity. The dotted lines show where the cut-off frequency is exactly $300 \mathrm{~s}$.

upwards into the rest of the solar atmosphere. Results from these simulations should provide further insight into the possible leakage of solar global 5 min photospheric $p$-modes into the solar chromosphere, transition region and corona, in regions where the non-magnetic approximation is reasonable.

The $300 \mathrm{~s}$ driver is applied at a height of $200 \mathrm{~km}$, below the photospheric temperature minimum. In the VALIIIc atmosphere this should be a region of decreasing temperature, but to suppress convective instability in this part of the domain the temperature distribution has been altered to be isothermal below the temperature minmum, at the minimum temperature. The driver period is close to and above the cut-off period at the point of application, and it is expected that the signal will be evanescent immediately above the driver height up to $\sim 700 \mathrm{~km}$, and therefore that the resonant excitation at the cut-off frequency will become the dominant signal.

The high period simulation has a somewhat richer behaviour than the low period, and the behaviour is continuously evolving with time. It is helpful to consider the behaviour of the signal along the central axis of the domain, reducing the analysis to 1D for a moment, by taking a time distance image along this axis, see Fig. 4. Note that on this image are marked dotted lines depicting the height at which the local cut-off period is exactly $300 \mathrm{~s}$. These delineate the region at the bottom of the domain in the upper photosphere and lower chromosphere in which the cut-off is $<300 \mathrm{~s}$, and the region around $2.2 \mathrm{Mm}$ at the transition region where the strong stratification reduces the cut-off period. In these regions any signal at $300 \mathrm{~s}$ should be evanescent, while between them and in the corona such a signal should be propagative. Where the "chromospheric cavity" is referred to, it should be taken to mean the propagative region in the mid to upper chromosphere between the first and second of these lines as one goes upwards in height. The driving signal can be seen clearly as a thin band of alternating bright and dark with the $300 \mathrm{~s}$ period at the bottom of the image. However, looking immediately above the driver height there is a signal with a slightly lower period with similar amplitude, which is first apparent after about $220 \mathrm{~s}$. This signal, at a higher than driven frequency, becoming stronger over time is a consequence of the resonant excitation of the cutoff frequency mode, and as predicted, given the evanescence of the higher period driven signal, it quickly becomes the dominant signal in the upper photosphere.
Moving further up into the atmosphere, it is apparent that at all heights the dominant behaviour is at a lower period than the driver period, but there is some local variation in this dominant behaviour. At the transition region for instance the dominant period is lower again than the photospheric period, while in the chromosphere the period is very marginally higher than in the upper photosphere. This region is discussed below in more detail in Sect. 6.2.

Figure $5 \mathrm{~b}$ which is the time distance image taken at a height of 3.3 Mm in the corona, shows (for the first $\sim 600 \mathrm{~s}$ in particular) that there is still some wave transmission beyond the transition region, but this transmission shows nothing like the strong amplitude growth from the driver amplitude which the stratification gives to the lower period waves. Rather, the maximum amplitude recorded at $3.3 \mathrm{Mm}$ is $\sim 120 \mathrm{~m} \mathrm{~s}^{-1}$ which is below the maximum amplitude at $1.3 \mathrm{Mm}$ of $130 \mathrm{~m} \mathrm{~s}^{-1}$ and the driver amplitude of $270 \mathrm{~m} \mathrm{~s}^{-1}$. This is a clear sign that wave energy is prevented from propagating higher, firstly by the resonant excitation of cutoff modes due to driver evanescence, and secondly by the strong reflection which results at the transition region. The transmission coefficient for these higher period signals is much lower than for low period signals. It is estimated that only of the order of $1 \%$ of the wave energy is able to penetrate from the chromospheric cavity to the corona. The first transmitted wavefront occurs after $150 \mathrm{~s}$, and is the first pulse from the driver. Quickly after this at $250 \mathrm{~s}$ is a second peak. It is clear from these timings that the second peak cannot have originated from the second driver peak, nor is there a reflection from a higher point in the corona. This coronal signal in fact originates from the resonant excitation of the transition region, as is visible in Fig. 4. The photospheric $p$-modes have thus generated a lower period signal (in this case $\sim 250 \mathrm{~s}$ ) which is transmitted into the corona. The behaviour visible in Fig. 5b is more complex than this however. After $1000 \mathrm{~s}$ in particular, there are several separate signals at different horizontal positions. The horizontal behaviour visible in Fig. 5b is due to the leakage into the corona of the transition region surface waves discussed below in Sect. 6.3. Higher in the corona these surface waves would not be apparent.

\subsection{Standing waves}

Figure 6 is a snapshot of vertical velocity in the domain after some time has passed.

Before examining the question of resonant excitation and the frequency composition of the signals above the driver, compare the behaviour of the amplitude here to the behaviour of the amplitude in the lower period case. While the lower period signals show immediate growth due to stratification, this is not apparent here. Rather, the signal is in fact apparently damped by evanescence up to $\sim 700 \mathrm{~km}$. In the upper chromosphere the signal should be propagative again (whether the dominant signal is at the driver or cut-off period) and here stratification driven growth does begin, although it is not so clearly displayed as in the lower period case. Nevertheless, the velocity does achieve a local maximum amplitude at the transition region as before, although now this is comparable to the driver amplitude, where in the low period case it was significantly greater.

There are several more features on this snapshot which are of interest. Going from left to right, from photosphere to corona on the plot of vertical velocity $v_{z}$ against height along the central axis of the computational domain the first feature is the driver. This is visible as the local minimum at the far left of the plot. Clearly, the driver is not in phase with the behaviour of the rest of 

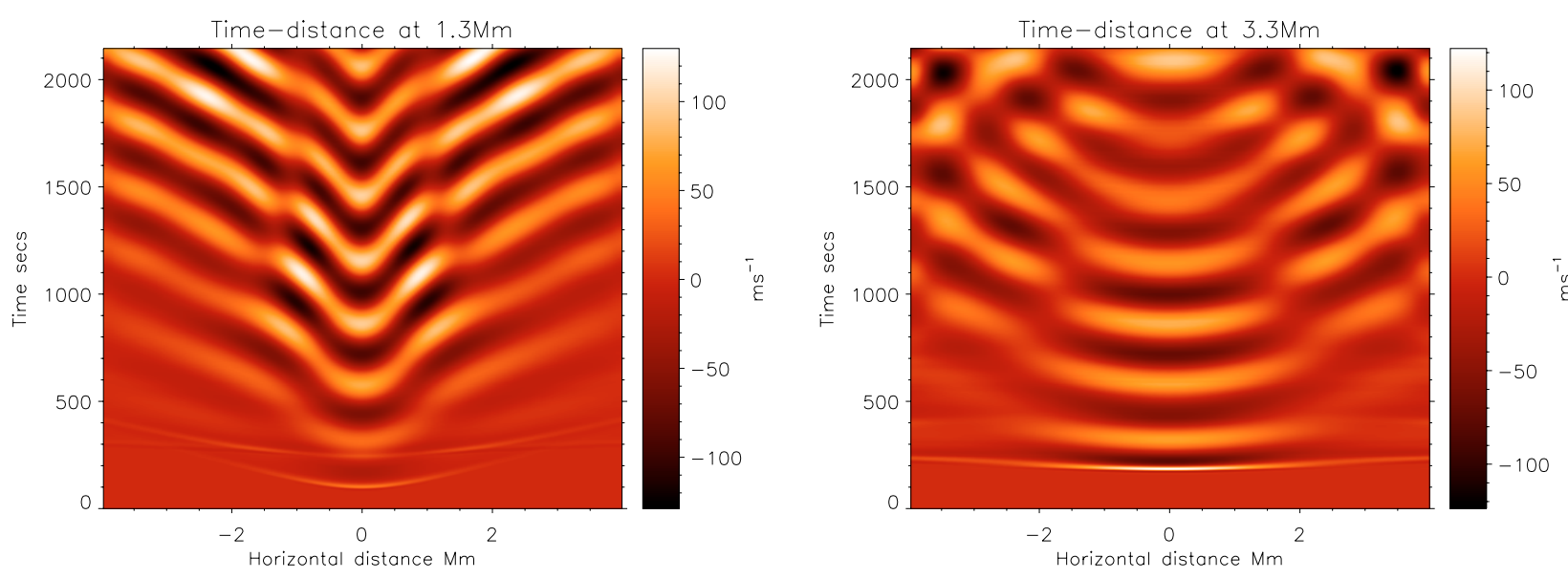

Fig. 5. a) Time-distance image for $300 \mathrm{~s}$ driver showing vertical velocity along a horizontal line taken at $1.3 \mathrm{Mm}$ into the atmosphere (in the chromosphere). b) Similar to a, but taken at a height of $3.3 \mathrm{Mm}$ and thus in the corona.
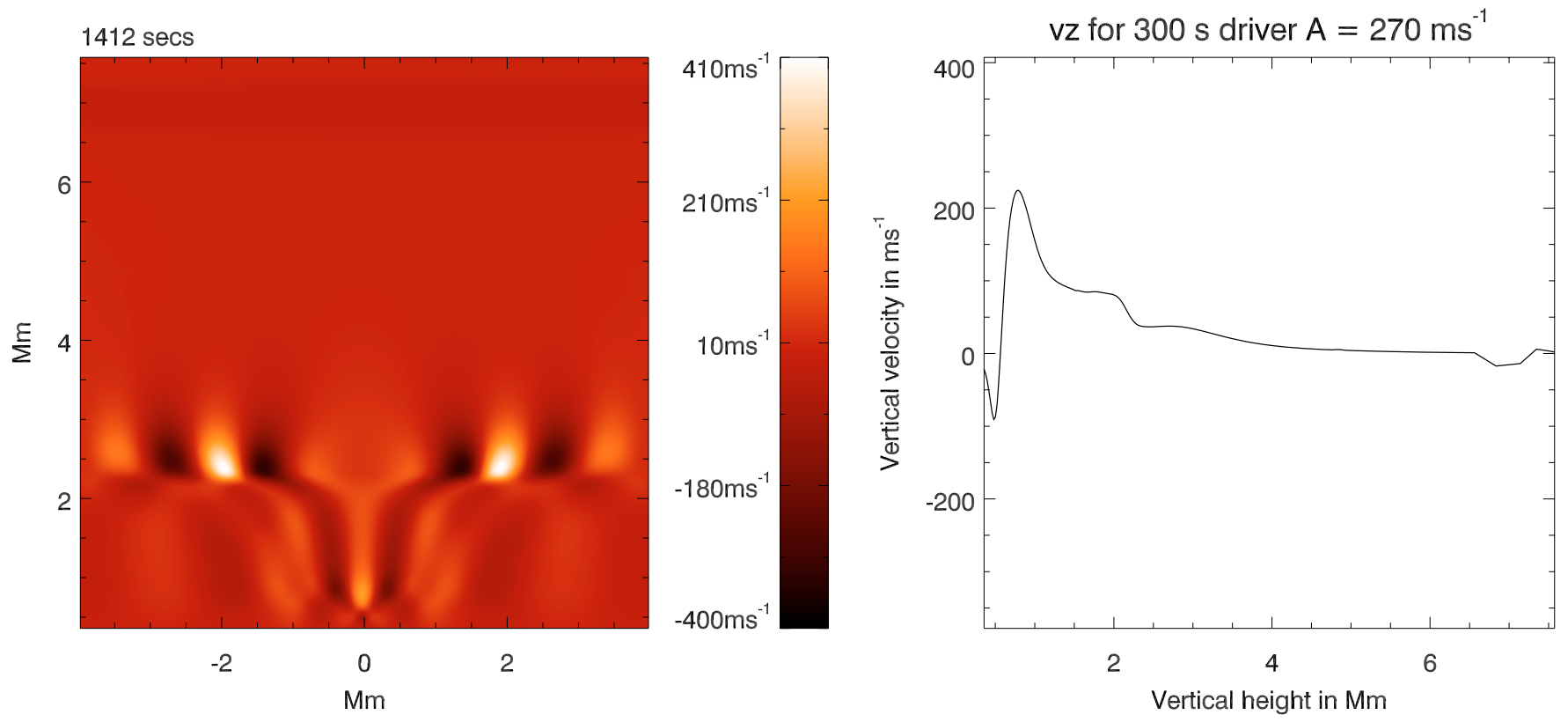

Fig. 6. Same as Fig. 2 but for a $300 \mathrm{~s}$ driver, snapshot taken after $1412 \mathrm{s.}$

the model atmosphere, registering as it does as the only negative velocity along this line.

Although the driver is not in phase, the signal from the upper photosphere above the driver right through to the transition region and corona is uniformly positive. Movies of the data support the impression that a standing mode or something very close to a standing mode is set up along the whole length of the domain above the driver. To determine whether this is truly a single coherent standing mode, consider Fig. 7.

In Fig. 7a plots of the velocity at every datapoint along the central vertical axis in the region in which there is evidence of the standing wave have been superposed. The thickness of the lines in the time direction is an indicator of the extent to which the signals at these various different heights are coherent. If they were perfectly coherent then while the amplitudes might vary, each signal would achieve maximum amplitude at the same time, and the zeros of velocity would all occur at exactly the same time. The "width" in time of the region where the various signals record zero velocity is therefore a measure of how out of phase the signals are. In Fig. 7a there is clearly a strong similarity between the phase of the signals at different heights. However the signals are certainly not exactly in phase, with a typical lag of $\sim 50 \mathrm{~s}$ between the first and last signals to reach zero velocity and a clear tendency for the signals to become more out of phase as time progresses.

Considering again Fig. 4 the interpretation suggests itself that there is not a pure standing wave from the low chromosphere all the way to the TR, but that there are sub-regions in which the signal is coherent and in which the standing wave description may apply. Specifically, firstly there is the signal in the lower chromospheric evanescent region discussed above which seems to be coherent over $\sim 300 \mathrm{~km}$ in height. Above this, in the propagative chromospheric cavity it appears that the signal is largely coherent over $\sim 800 \mathrm{~km}$. In Fig. 4 this gives the appearance of light and dark stripes in the upper chromosphere, and these seem to occur at a period above the lower chromospheric period, but still lower than the $300 \mathrm{~s}$ driver period. Finally in the transition region it appears that the signal is relatively coherent over $\sim 400 \mathrm{~km}$. Figures $7 \mathrm{~b}-\mathrm{d}$ show the signals only at gridpoints in the low chromosphere, chromospheric cavity and transition 

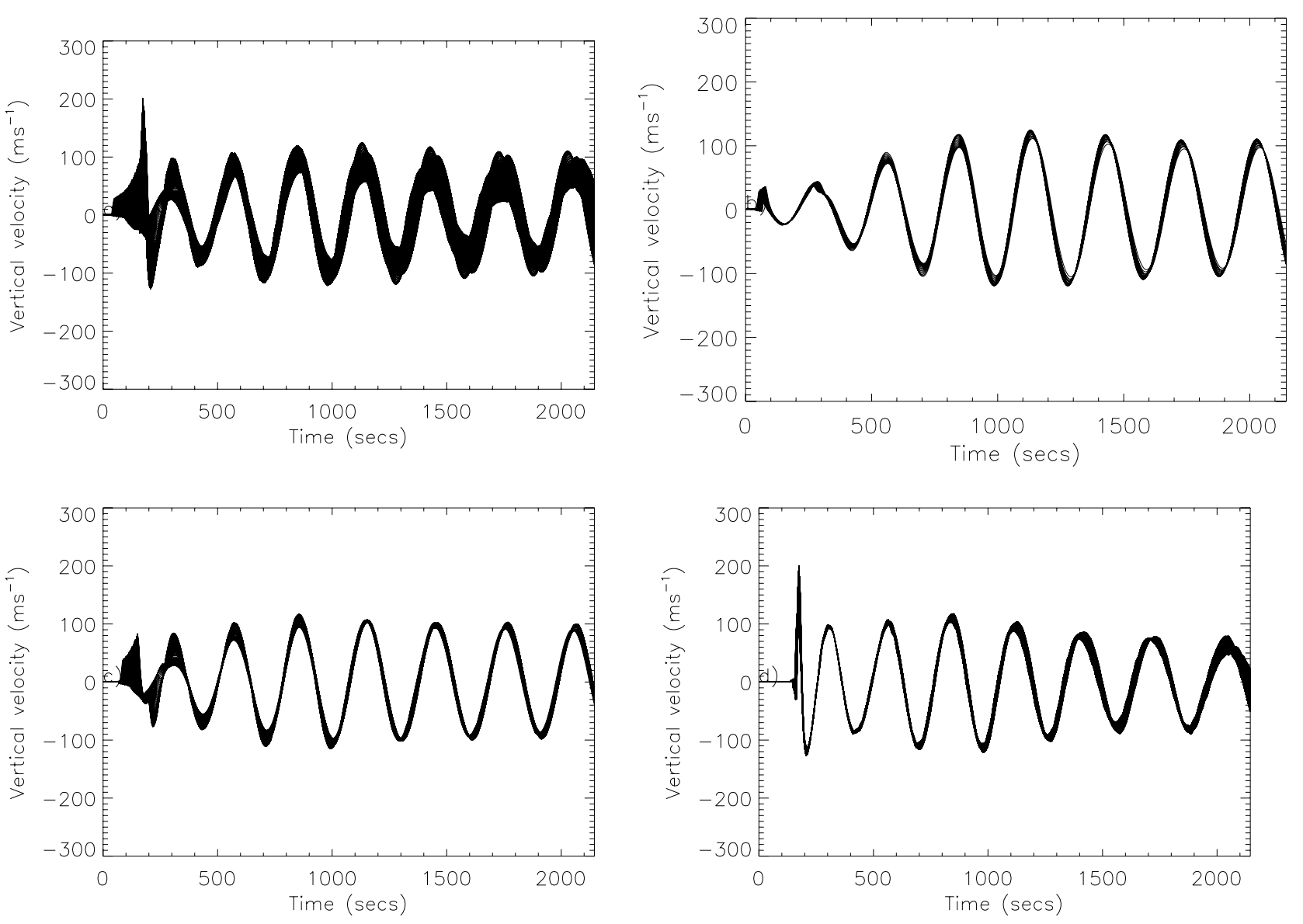

Fig. 7. a) Here the vertical velocity along the central vertical axis of the computational domain is plotted on the same axes for each gridpoint in the range $650 \mathrm{~km}<z<3.3 \mathrm{Mm}$. b) Similar to a, but in the range $700 \mathrm{~km}<z<1 \mathrm{Mm}$. c) Similar to a), but in the range $1.1 \mathrm{Mm}<z<1.9 \mathrm{Mm}$. d) Similar to a), but in the range $2.1 \mathrm{Mm}<z<2.5 \mathrm{Mm}$.

region respectively. It is clear that in each of these regions, the signals are indeed very strongly coherent, while looking at Fig. 8 which shows the signal at a single data point in each region it is equally clear that there is a difference in phase and frequency between these signals.

It is suggested based on the above analysis that there is not a single standing wave behaviour of the whole chromosphere and TR, but rather three separate standing phenomena oscillating with very similar but not identical periods.

Returning to Fig. 6 the next feature moving to the right from the driver, is a local maximum at about $750 \mathrm{~km}$. This is the area of the lower chromosphere above the temperature minimum in which the local acoustic cut-off frequency is $>3.3 \mathrm{mHz}$. It is expected (Fleck \& Schmitz 1991; Rae \& Roberts 1982; Roberts 2004) that a signal below the cut-off frequency will cause resonant excitation, and this fits with the results. This local maximum of amplitude occurs at the height at which resonance becomes dominant over the driven signal. The signal at this height starts to oscillate at the local cut-off period of $\sim 280 \mathrm{~s}$, but by the end of the simulation is oscillating with a period of $300 \mathrm{~s}$. This supports the theoretical prediction (Fleck \& Schmitz 1991) that the driver period should become dominant as time progresses. This resonantly excited signal is the coherent signal in the lower chromosphere from Fig. $7 \mathrm{~b}$.

The next part of the graph, from 1-2 Mm, is harder to interpret. The forward modelling data show clear evidence of the existence of a coherent standing wave in the chromospheric cavity,

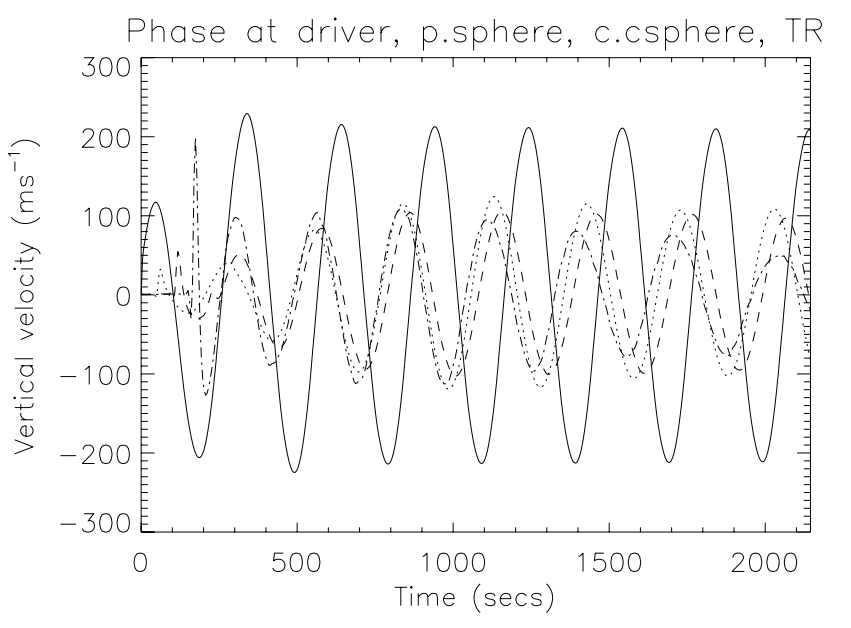

Fig. 8. The four lines on this graph plot the vertical velocity at four different heights on the central axis. The solid line is at the driver height, the dotted line is above the driver in the lower chromosphere where the $300 \mathrm{~s}$ signal is evanescent, the dashed line is in the chromospheric cavity and the dot-dashed line is in the transition region.

see Fig. 7c. Fleck \& Deubner (1989) presented observational evidence of the existence of standing waves in the chromosphere, and thus the presence of standing waves in the simulations is of great interest. In Fig. 6 in the 2D image the vertically 
coherent waves spreading outwards from the centre at chromospheric heights suggest that there is a standing mode in the cavity, and this can be seen in Fig. 4 where the alternating light and dark bars in the chromospheric region 1-2 Mm show that the signal is approximately vertically coherent. The signal here starts with a period of about $255 \mathrm{~s}$, but this increases to $300 \mathrm{~s}$ (never above $300 \mathrm{~s}$ ) by the end of the simulation.

In this region, the $300 \mathrm{~s}$ signal, or for that matter any resonant signals from lower, should be able to propagate. Any resonant response at the local cut-off frequency should therefore be at a period $>300 \mathrm{~s}$, but should also be negligible compared to the propagating signal. Rather, the presence of what seems to be a highly coherent standing wave in this region is due to reflection of the propagating signal at the transition region, at a height of $\sim 2.1 \mathrm{Mm}$ (Fig. 4). There may also be reflection at the bottom of the chromospheric cavity, however the stratification here is very much gentler than at the transition region and therefore any reflection is likely to be weak. In Fig. 6 there is a local maximum at about $2 \mathrm{Mm}$ followed by an inflection at about 2.2 $\mathrm{Mm}$ which is an indicator of the division between the cavity mode and the transition region resonant excitation. The transit time from the bottom of the chromosphere to the reflection point and back again is $\sim 270 \mathrm{~s}$, and this is a good match for the period of the chromospheric standing wave as would be expected. The reflection based interpretation of the standing mode does not offer an explanation of the increase in signal period from 255 to $300 \mathrm{~s}$. However, given that the chromospheric cavity is only semi-closed it is unsurprising that the behaviour of the resonant signal, especially below, influences the cavity signal. There is further discussion of the cavity mode in Sect. 6.4.

Attempts were undertaken to model analytically this chromospheric standing mode as a wave on a string, and in 1D using the Klein-Gordon equation, but it was repeatedly found that analytical results did not match the simulated results well enough. Part of the problem may be that the resonant signals above and below the cavity mask what would otherwise be the nodes of a standing wave, in particular at the transition region. Also, a problem reflecting the complexities of the model used in the simulations would make it extremely difficult to solve the KleinGordon equation, whereas over-simplifying renders the results irrelevant. While there may be further potential to mirror some of these results analytically, the task is considered therefore to be beyond the scope of this paper.

Finally, from about $2.1-3.5 \mathrm{Mm}$, is the transition region signal. On the graph snapshot (Fig. 6), it is visible to the right of the local maximum around $2 \mathrm{Mm}$. On the full 2-D image however this signal is very clear, manifesting as the centre of a surface wave propagating out along the transition region. Figure 4 clearly shows that this signal has a higher maximum amplitude than that of the chromospheric standing wave, but an amplitude below that of the resonantly excited wave in the photosphere or of the driver. Comparing this to the result for the high frequency $30 \mathrm{~s}$ wave for which the transition region represented maximum amplitude, it is evident that much of the wave energy fails to reach the transition region, instead being trapped in the photosphere and chromosphere. The signal initially has a period of $\sim 235 \mathrm{~s}$, but this increases to $270 \mathrm{~s}$ and thence to $300 \mathrm{~s}$ by the end of the simulation. The signal is due to the resonant excitation of the transition region cut-off frequency mode, and as above this increase to the driver period is predicted analytically. In the transition region the cut-off period is strongly varying, and drops significantly lower than $235 \mathrm{~s}$. However, it seems that a response is excited primarily at a "typical" cut-off and that within the transition layer the signal is largely coherent despite this variation, see Fig. 7.

The simulations produce three standing signals at very similar periods, which start off approximately in phase. The superposition of these signals therefore forms a quasi-standing mode over about $1000 \mathrm{~s}$, during which period they are close enough to being in phase that they could easily be interpreted as a single signal. Indeed, given that $p$-modes are only observed to be coherent over about 6 periods at any given point, it seems plausible that observations might give the appearance of a single phenomenon where in fact there may be more than one mechanism at work. It is suggested that whereas previous work (Fleck \& Deubner 1989; Fleck \& Schmitz 1991; Leibacher et al. 1982) has attempted to explain standing modes and $200 \mathrm{~s}$ signals observed in the chromosphere as either generated by partial reflection at the transition region, or as the result of resonant excitation at the cut-off frequency, it is more appropriate to consider both phenomena as important, and as able to generate oscillatory signals at similar amplitudes.

\subsection{Transition region guided waves}

Returning to the full 2D behaviour of the simulation, Fig. 5a displays the propagation of the signal across a line at $1.3 \mathrm{Mm}$ height, and shows the behaviour of the signal in the horizontal dimension in the chromosphere. The shape of the wavefronts at this height is much more time dependent than in the lower period case. The first two wavefronts detected at this height (within $\sim 700 \mathrm{~s}$ ) show the curvature of upwards propagating waves at the centre of the domain. The first front is very narrow and quite faint on this image, impacting on $1.3 \mathrm{Mm}$ after slightly less than $100 \mathrm{~s}$, and has uniform direction of curvature, indicating that the pulse caused by the first wavefront travels up through the atmosphere as one might expect. Just above this front, and below the first "thicker" signal, is the flatter signature of the first reflected wave travelling back down the simulation from the transition region.

Above this at about $300 \mathrm{~s}$ is a much thicker wavefront. In the centre (about $\pm 0.7 \mathrm{Mm}$ ) the strongest part of this signal seems to have a strong curvature which suggests an upwardly propagating signal. Closer examination reveals that there is a faint wave front very similar to that of the first pulse, which is a simple upwardly propagating front. The dominant signal however is the horizontal propagation of a signal outwards from the centre. Figure 6 shows these outward propagating waves, which are slanted due to the increasing sound speed. This signal is launched at the same time that the second upward wave is detected, and superposed in the centre,but has much greater amplitude because the standing wave is set up from this point on. In the chromosphere this signal is damped as it travels outwards, and the amplitude is greatly reduced beyond $\pm 2 \mathrm{Mm}$. However, at the transition layer the speed is greater and the transition region acts as a guide and the wave on this surface has a strong amplitude and is still strong when it leaves the domain at $\pm 4 \mathrm{Mm}$.

The transition region wave and the chromospheric standing wave start in phase and thus these horizontally moving wavefronts start almost coherent from chromosphere to transition. However, the transition region signal is of a higher frequency, and therefore the transition region wave becomes out of phase with the lower signal over time. This generates some complex behaviour at the interface between the chromospheric cavity and the transition layer, but it does not seem to be of crucial importance to the behaviour of the system. 

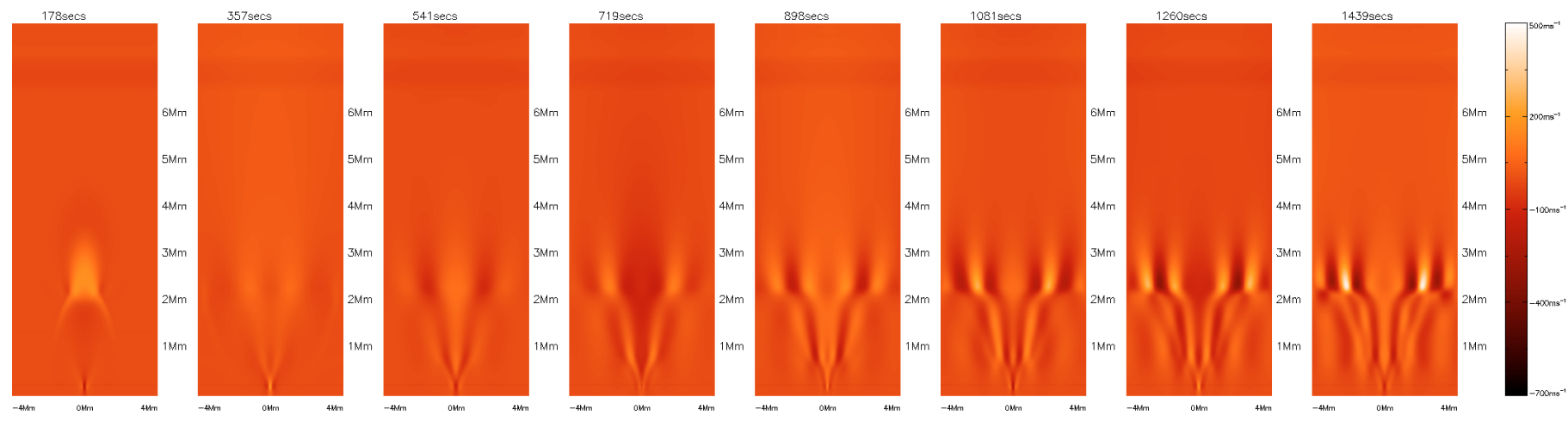

Fig. 9. Time series of images showing the development of a guided wave at the transition region from a $300 \mathrm{~s}$ driven signal.

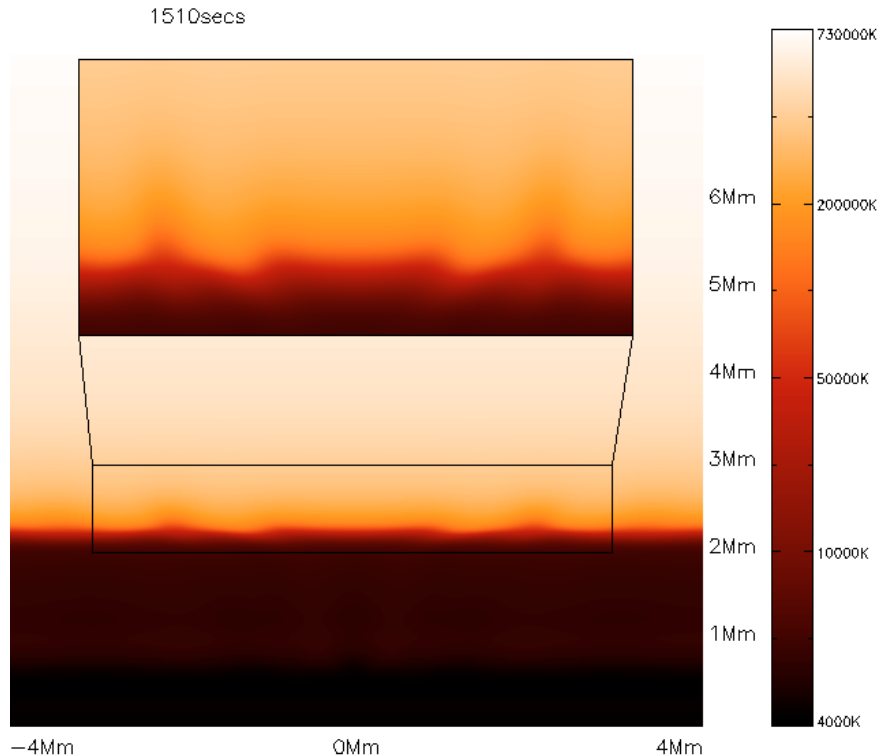

Fig. 10. Snapshot of the log of the temperature in the computational domain for a simulation driven with an amplitude of $760 \mathrm{~ms}^{-1}$, zoomed in to show the development of fine structure rising from the transition region, taken after $1510 \mathrm{~s}$.

Figure 9 allows us to clearly see the development of this guided wave, and also makes clear the strong amplitude of these guided waves compared to the velocity signals elsewhere in the domain. The transition region guided wave has a wavelength of $\sim 160 \mathrm{~km}$ and the period of the waves is around $300 \mathrm{~s}$, but there is a strong variation with the period at different times and positions within the transition region varying in a range $\sim 250-350 \mathrm{~s}$.

This guided wave (which is a vertical velocity wave) causes a small lifting of cool dense material into the corona. At the $270 \mathrm{~m} \mathrm{~s}^{-1}$ driver amplitude this movement is rather insignificant, but in runs performed at higher amplitudes for comparison the phenomenon becomes more noticeable, for instance in Fig. 10 where there are dark protrusions rising $\sim 200 \mathrm{~km}$ above the equilibrium height of the transition region, driven by a $760 \mathrm{~m} \mathrm{~s}^{-1}$ amplitude signal. Although such harmonic amplitudes are unphysical at a single point, this does suggest that if a little more energy were put into the system (by driving the whole lower boundary for instance or by driving at more than one point) that more interesting transition region behaviours might be found, which should be addressed in a future paper. The behaviour of the transition region is also somewhat richer in the cold chromosphere simulations, see Sect. 6.5.

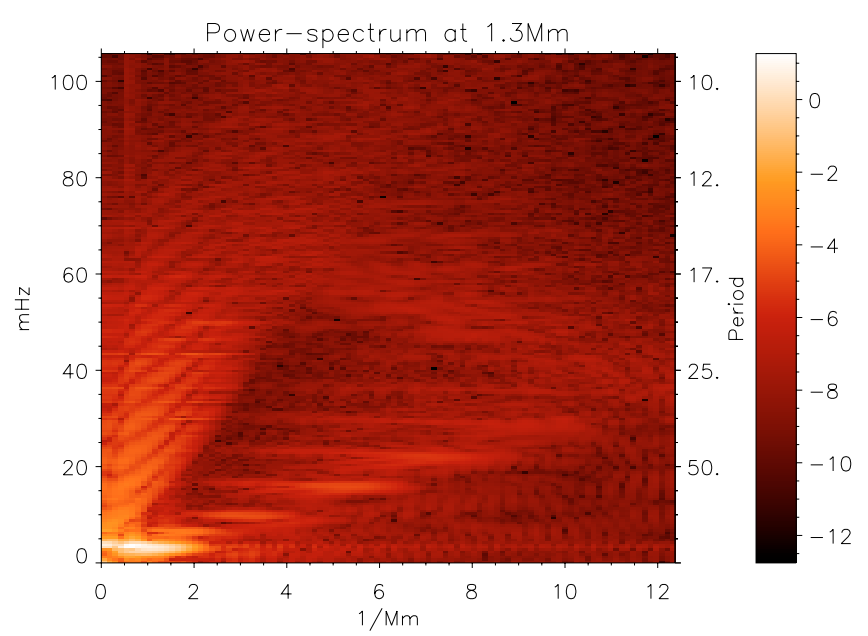

Fig. 11. Power spectrum for the case with a $300 \mathrm{~s}$ driver taken along a horizontal cross-section at $1.3 \mathrm{Mm}$ height.

An initial effort has been made to find a dispersion relation which would describe the properties observed in this guided wave, by considering the dispersion equation in e.g. Miles \& Roberts (1992) for a two isothermal layer atmosphere and also considering a simple three layer atmospheric model. Neither of these models proved to be a sufficiently close approximation to the realistic simulated atmosphere that the equations were satisfied, or close to being satisfied. A full theoretical consideration of the dispersion relation for these guided waves is again somewhat outside the scope of this paper, but will be addressed in another more mathematically inclined study.

\subsection{Cavity modes}

Figure 11 is the power spectrum within the chromospheric cavity generated by the $300 \mathrm{~s}$ driver. It clearly reveals the signature of the standing modes being set up in the cavity, in the form of localised areas of high signal power, with the driven power showing up clearly at $\sim 3.3 \mathrm{mHz}$ and higher harmonics at $\sim 6.5,10,16$ and $22.5 \mathrm{mHz}$. The frequencies are close to the harmonics of the 5 min signal, but the power spectrum does not resolve the frequencies well enough to distinguish clearly between the key signal periods mentioned above. These results are consistent with the presence of a standing signal between 250 and $300 \mathrm{~s}$ in period, but would not distinguish between two different signals at such close frequencies. The response at 13.3 and $20 \mathrm{mHz}$ seems somewhat weaker, but is still visible on the spectrum, and as the 


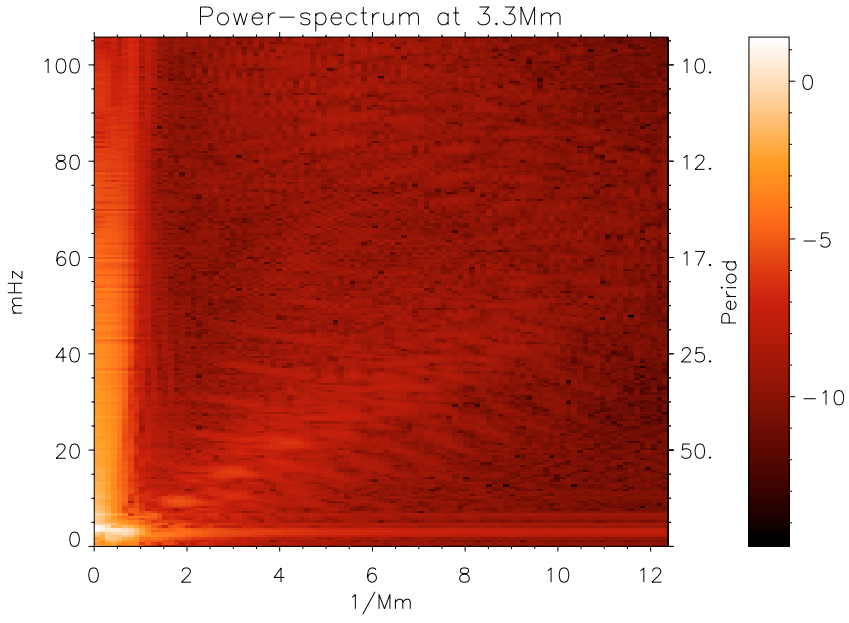

Fig. 12. Power spectrum for the case with a $300 \mathrm{~s}$ driver taken along a horizontal cross-section at $3.3 \mathrm{Mm}$ height.

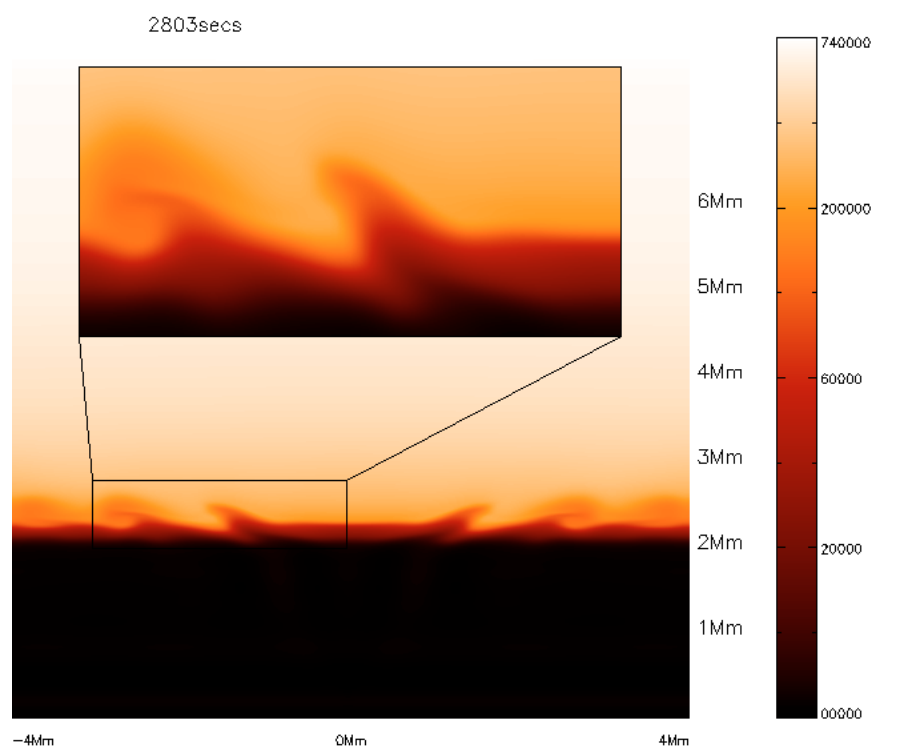

Fig. 13. Snapshot of the log of the temperature from the cold chromospheric case with a $300 \mathrm{~s}$ driver, with a section of the transition region expanded to show small cold protrusions rising towards the low corona.

sound speed is constant along the cross-section along which the spectrum is taken, the localised power signals of the harmonics are in a straight line. The equivalent power spectrum taken at a position above the transition region (Fig. 12), contains only evidence of the driven $300 \mathrm{~s}$ signal, and at a lower power something of the first harmonic signal. There is nothing to generate harmonics from the fundamental signal above the transition region, rather it is evidence of leakage of power at these frequencies from the chromosphere.

\subsection{Cold chromospheric case}

For the reasons mentioned earlier, further $300 \mathrm{~s}$ driven simulations have been produced using a temperature model which assumes a cold isothermal chromosphere. Most of the key results obtained are not fundamentally changed by the alternative temperature structure, however there is an important alteration in the behaviour at the transition region. In the cold chromospheric case the start of the transition region is more abrupt than in the

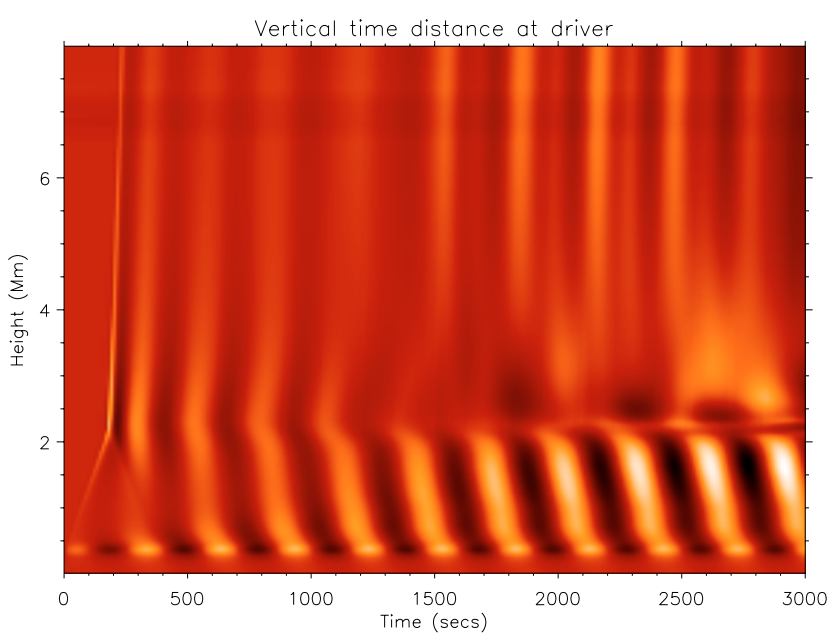

Fig. 14. Time distance image along the central vertical axis of the computational domain showing vertical velocity.

hot chromosperic VALIIIc, and it was found that this caused the transition region to act more effectively as a reflective surface for the upwards propagating signals. A node is formed at the reflection height directly above the driver, and the resonant transition region signal is severely limited in the centre of the domain, with the chromosphere behaving very much as a semi-open cavity. More energy is channelled into the surface waves which drive structured protrusions into the corona, as can be seen in Fig. 13.

There is no variation of cut-off period in the chromosphere now, so the lower chromospheric resonance and the cavity mode are at the same frequency, see Fig. 14, and this differs from the frequencies observed in the VALIIIc case. The important conclusion to be drawn from these additional results is that overall the simulations are not dramatically different from the VALIIIc results, and demonstrate that although the system is sensitive to the temperature structure in the chromosphere, it is not so sensitive that conclusions based on one model cannot be relevant to another. Nevertheless, they also show differences in behaviour which are sufficiently interesting to suggest that the question of how the detail of chromospheric temperature structure affects $p$ mode propagation is an interesting one which deserves further study.

\section{Summary}

Simulations of the propagation of driven waves from the upper photosphere into the chromosphere and corona (in a nonmagnetic case) have been carried out, and have resulted in three key results.

Firstly, the simulations have shown the strong propagation of waves driven at frequencies higher than the transition layer cut-off frequency into the corona, with relatively low reflection at the transition region.

Secondly, due to both resonant excitation of the atmosphere at the cut-off frequency and partial reflection of upwards propagating signals at the chromosphere-transition region boundary, the $300 \mathrm{~s}$ driver is able to set up standing waves in the chromospheric cavity, and at the photosphere and transition region. This is also true of drivers at similarly high periods from $250-350 \mathrm{~s}$, although where the driver is more out of phase with the resonant excitations the standing waves are less clearly defined, and in the case of a driver of $450 \mathrm{~s}$, for instance, the three signals become completely out of phase with each other over only $\sim 4$ periods. 
Thirdly, for the 5 min driven signal, but also for all the other drivers which have been considered (although not discussed in detail here) with periods in the range 200-400 s, the signals drive the formation of transition region guided waves propagating horizontally, with periods around $300 \mathrm{~s}$ and wavelength of around $160 \mathrm{~km}$. This guided wave formation is of particular interest, and we intend to extend the work to a more realistic model with the aim of determining what observational signatures might be associated with such guided waves.

For all driver amplitudes we have found that the simulated waves experience strong stratification driven growth to a local maximum at the transition region, which is in good agreement with the results of various observational studies of three minute sunspot oscillations, which have found that the observed oscillation amplitude of such waves above sunspots peaks at transition region temperatures (Brynildsen et al. 2002; Fludra 2001; Marsh \& Walsh 2006; O'Shea et al. 2002).

With the further investigation of the transition region guided waves as a key priority, we expect interesting results to be yielded by considering driving the full extent of the lower boundary at realistic photospheric velocities, including a realistic quiet Sun magnetic field geometry and looking at including a simple form of radiative emission.

Acknowledgements. The authors thank Dr Y. Taroyan for a number of useful discussions. R.E. acknowledges M. Kéray for patient encouragement. C.M. acknowledges the support of C. Whalley. The authors are also grateful to NSF, Hungary (OTKA, Ref. No. TO43741) and the Particle Physics and Astronomy Research Council (PPARC), UK for the financial support they received.

\section{References}

Avrett, E. H. 1996, in Stellar Surface Structure, ed. K. G. Strassmeier \& J. L. Linsky, IAU Symp., 176, 503

Ayres, T. R. 1998, in New Eyes to See Inside the Sun and Stars, ed. F.-L.

Deubner, J. Christensen-Dalsgaard, \& D. Kurtz, IAU Symp., 185, 403

Ayres, T. R. 2002, ApJ, 575, 1104

Bogdan, T. J. 2000, Sol. Phys., 192, 373

Bogdan, T. J., Carlsson, M., Hansteen, V. H., et al. 2003, ApJ, 599, 626
Brynildsen, N., Maltby, P., Fredvik, T., \& Kjeldseth-Moe, O. 2002, Sol. Phys., 207, 259

Carlsson, M. \& Stein, R. F. 1992, ApJ, 397, L59

Carlsson, M. \& Stein, R. F. 1995, ApJ, 440, L29

Carlsson, M. \& Stein, R. F. 2002, in SOLMAG 2002 Proceedings of the Magnetic Coupling of the Solar Atmosphere Euroconference, ed. H. SawayaLacoste, ESA SP-505, 293

Chaouche, L. Y. \& Abdelatif, T. E. 2005, Sol. Phys., 229, 255

De Moortel, I. \& Hood, A. W. 2003, A\&A, 408, 755

De Moortel, I. \& Hood, A. W. 2004, A\&A, 415, 705

De Moortel, I., Ireland, J., Hood, A. W., \& Walsh, R. W. 2002a, A\&A, 387, L13

De Moortel, I., Ireland, J., Walsh, R. W., \& Hood, A. W. 2002b, Sol. Phys., 209, 61

De Pontieu, B. 1996, Ph.D. Thesis

De Pontieu, B., Erdélyi, R., \& de Wijn, A. G. 2003, ApJ, 595, L63

De Pontieu, B., Erdélyi, R., \& James, S. P. 2004, Nature, 430, 536

De Pontieu, B., Erdélyi, R., \& De Moortel, I. 2005, ApJ, 624, L61

Fleck, B. \& Deubner, F.-L. 1989, A\&A, 224, 245

Fleck, B. \& Schmitz, F. 1991, A\&A, 250, 235

Fludra, A. 2001, A\&A, 368, 639

Fontenla, J. M., Avrett, E. H., \& Loeser, R. 1990, ApJ, 355, 700

Fossum, A. \& Carlsson, M. 2005, Nature, 435, 919

Hasan, S. S., van Ballegooijen, A. A., Kalkofen, W., \& Steiner, O. 2005, ApJ, 631,1270

James, S. 2004, Ph.D. Thesis, University of Sheffield

Kalkofen, W. 2001, ApJ, 557, 376

Kalkofen, W., Rossi, P., Bodo, G., \& Massaglia, S. 1994, A\&A, 284, 976

Kalkofen, W., Ulmschneider, P., \& Avrett, E. H. 1999, ApJ, 521, L141

Lamb, H. 1932, Hydrodynamics (New York: Dover Publ.)

Leibacher, J., Gouttebroze, P., \& Stein, R. F. 1982, ApJ, 258, 393

Marsh, M. S. \& Walsh, R. W. 2006, ApJ, 643, 540

McWhirter, R. W. P., Thonemann, P. C., \& Wilson, R. 1975, A\&A, 40, 63

Miles, A. J. \& Roberts, B. 1992, Sol. Phys., 141, 205

O'Shea, E., Muglach, K., \& Fleck, B. 2002, A\&A, 387, 642

O'Shea, E., Banerjee, D., \& Doyle, J. G. 2006, A\&A, 452, 1059

Rae, I. C. \& Roberts, B. 1982, ApJ, 256, 761

Roberts, B. 2004, in Oscillations and Small-Scale Transients Events in the Solar Atmosphere: Joint View from SOHO and TRACE, ed. R. Erdélyi, J. Ballester, \& B. Fleck, ESA SP-547, SOHO 13 Waves, 1

Schmitz, F. \& Fleck, B. 1998, A\&A, 337, 487

Sutmann, G., Musielak, Z. E., \& Ulmschneider, P. 1998, A\&A, 340, 556

Tóth, G. 1996, Astrophysical Letters Communications, 34, 245

Vernazza, J. E., Avrett, E. H., \& Loeser, R. 1981, ApJS, 45, 635

Zhukov, V. I. 2002, A\&A, 386, 653 